\title{
SIMULATOR TRAINING IMPROVES DRIVER EFFICENCY: TRANSFER FROM THE SIMULATOR TO THE REAL WORLD
}

\author{
David L. Strayer \& Frank A. Drews \\ Department of Psychology \\ 380 S. 1530 E. Rm 502 \\ University of Utah \\ Salt Lake City, UT 84112, USA \\ E-mail: David.Strayer@utah.edu
}

\begin{abstract}
Summary: Here we report the results of a fuel management simulation study to quantify the improvement in fuel efficiency for CDL truck drivers. Forty drivers were selected from a local commercial trucking company that maintained precise records on drivers' history, fuel efficiency, type of vehicles driven, and trucking routes. These drivers participated in a two-hour training program that focused on ways to optimize shifting to maximize fuel efficiency (e.g., progressive shifting, double clutching, timing, and appropriate gear selection). Transfer of training was assessed over a six-month interval using measures of fuel consumption obtained by drivers in their own vehicles driving their normal route. Training increased fuel efficiency by an average of $2.8 \%$ over the six-month interval. Analyses indicated that the benefits of training persisted throughout the posttraining interval. These training benefits were obtained even for the subset of drivers who changed vehicles after training, indicating that drivers learned a general skill that transferred from one vehicle to another. Additional analyses focused on which drivers benefited the most from training. We sorted the drivers into one of four groups, based on pre-training fuel efficiency. Our analysis indicated that those drivers with the lowest pre-training fuel efficiency benefited most from training (with over 7\% improvement in fuel efficiency), while those with the highest pre-training fuel efficiency did not benefit significantly from training. Together, our data validated the transfer of simulator training to realworld driving, as drivers incorporated the methods of optimal shifting into their driving practices. Moreover, the benefits of training appear to be durable and tend to benefit most those drivers whose performance was initially below the median on fuel efficiency.
\end{abstract}

Safety, maintenance, and fuel economy play crucial roles in the success of truck fleet operation. High-fidelity simulation offers the potential for providing a cost-effective and safe method for improving drivers' performance. But to provide effective training, it is important to identify the crucial elements to include in simulation and to determine the effectiveness of the training in terms of the transfer of acquired skills from the simulator to the real world. Here we report the results of a simulation study performed to quantify the improvement in fuel efficiency for CDL truck drivers. 


\section{METHOD}

Participants. Forty employees of a local trucking company participated in the study. All participants had a commercial drivers license (CDL), were between the ages of 25 and 66 (mean $=46$ ), and had worked for the company between 1 and 25 years (mean $=5$ ).

Stimuli and Apparatus. A TranSim VS ${ }^{\mathrm{TM}}$ simulator manufactured by GE Driver Development was used for simulator training. Detailed specifications on the simulator, pictured on the right, can be found on the GE Driver Development web site (http://www.cefcorp.com/i-sim/transimvs.htm).

Procedure. Drivers completed a 2-hour fuel management training course developed and delivered by GE Driver Development. The course was comprised of $19 \%$ lecture, $24 \%$ computer-based training, and $57 \%$ simulator training. Simulator training was

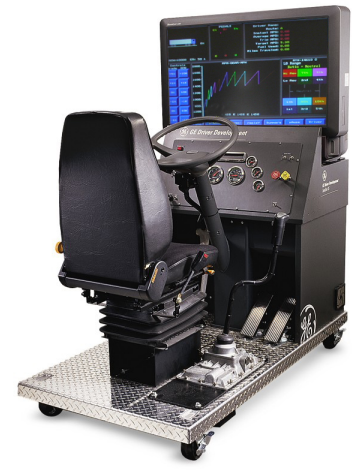
administered on the GE TranSim VS ${ }^{\mathrm{TM}}$ simulator. The training program focused on ways to optimize shifting to maximize fuel efficiency (e.g., progressive shifting, double clutching, timing, and appropriate gear selection). Following training, fuel consumption data for each driver was collected on a monthly basis over a 6-month interval.

\section{RESULTS AND DISCUSSION}

Figure 1 presents both the pre-training and 6month aggregated post-training fuel efficiency data measured by miles driven per gallon of fuel consumed. Overall, simulator training increased fuel efficiency by an average of $2.8 \%$. Statistical analysis indicated that the improvement was significant, $\mathrm{F}(1,39)=14.23$, $\mathrm{p}<.01$, establishing that the 2-hour fuel management program delivered by GE Driver

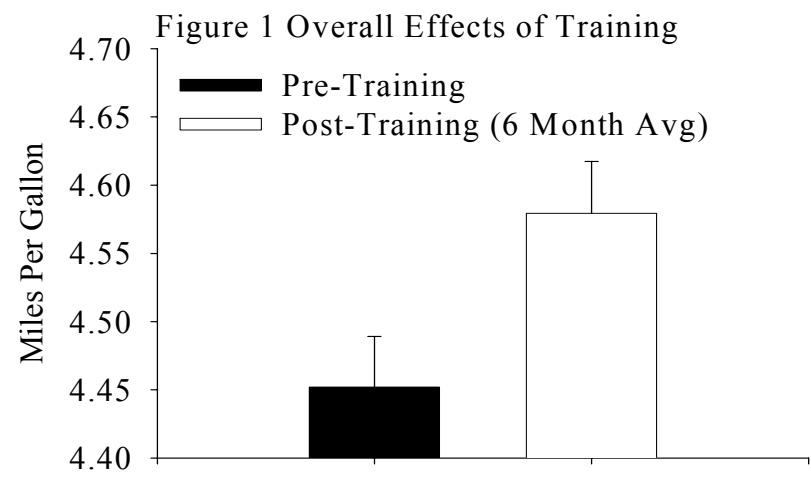
Development transfers to the real world, and improves CDL truck drivers' performance on this important dimension. However, because the omnibus analysis collapses over the 6-month interval for drivers of all skill level, this analysis does not provide information concerning how long-lasting the training effects are, or which drivers benefit most from training. Therefore, we performed a series of subsidiary analyses to determine how well the training was retained over the 6-month interval and how training affected drivers with different levels of pre-training expertise.

Figure 2 presents fuel consumption data for the pretraining period and for the 6 months of post-training data. It is evident from the figure that the effects of training persisted throughout the 6-month posttraining period. Statistical analysis indicated that the

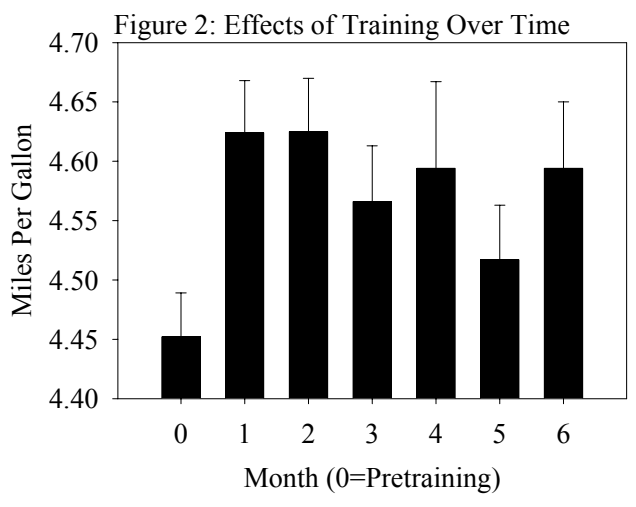


improvement for each post-training month was significant (all p's $<.05$ ). Thus, these data indicate that the effects of training are relatively long-lasting. Nevertheless, there is an apparent trend suggesting that the effects of training diminish over the 6-month post-training period. To estimate the level of skill retention over time, we converted the post-training data to percentages relative to pre-training baseline levels.

Figure 3 presents the post-training data referenced to pretraining baseline levels. Transfer of training scores greater than 0 indicate positive transfer from the fuel management program to the real world. Transfer was approximately $+4 \%$ for the first two months post-training. Thereafter, there was a modest decline in transfer, suggesting that some portion of the drivers may have reverted to pre-training habits (or alternatively, all drivers were slowly reverting to pre-training levels). The bestfitting power function, accounting for $40 \%$ of the

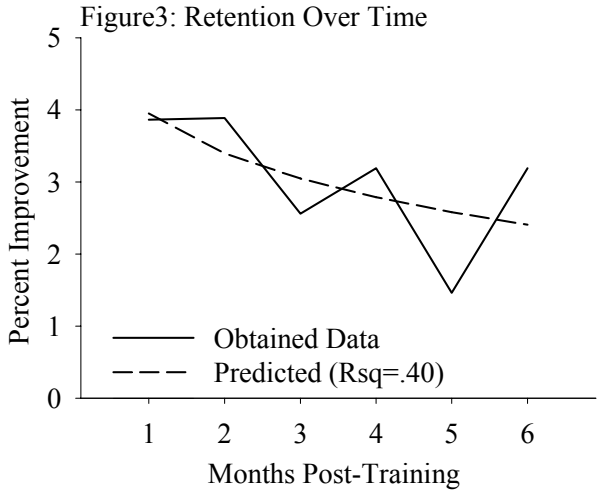
variance, indicated that transfer after 6 months was approximately $+2.5 \%$.

To determine which drivers were benefiting most from training, we divided the sample of 40 drivers into quartiles based on their pre-training fuel efficiency. Figure 4 presents the pre- and post-training fuel efficiency data for the four quartiles. As would be expected given the sorting strategy, pre-training fuel efficiency decreased systematically from the first to the fourth quartile. Inspection of the figure also reveals that not all drivers benefited equally from training. In particular, drivers in the first and second quartiles did not benefit significantly from the fuel management

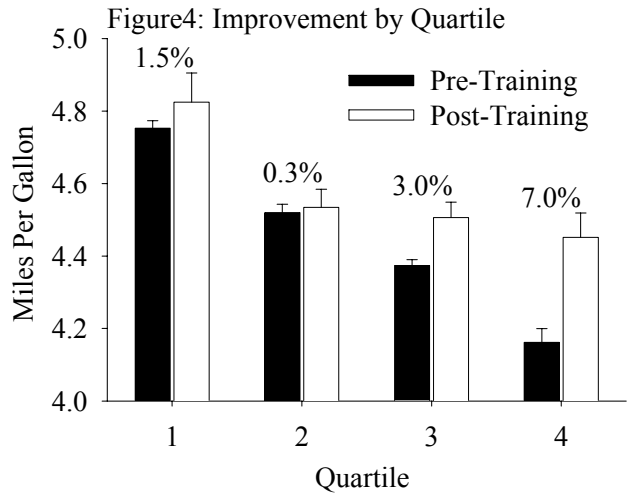
training, (p's > .30), due, at least in part, to ceiling effects. By contrast, drivers in the third and fourth quartiles benefited significantly from training $(\mathrm{t}(10)=2.6, \mathrm{p}<.05$; and $\mathrm{t}(10)=4.5, \mathrm{p}<.01$, respectively). That is, the drivers with the poorest pre-training fuel efficiency benefited most from the fuel management training program.

Given that drivers' pre-training performance was a determining factor in whether or not they benefited from the fuel management program, we performed a follow-up analysis that examined how the effects of training persisted across the 6-month interval for each of the quartile groups. Figure 5 presents the post-training data references to pre-training levels. As in Figure 3, transfer of training scores greater than 0 indicate positive transfer from the fuel-management program to the real world. Although the data are somewhat noisy given that there

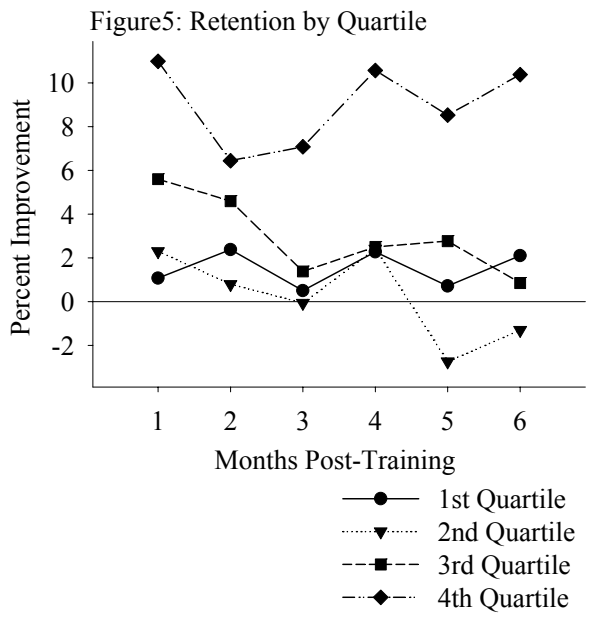
are only 10 subjects in each of the groups, it is clear that drivers in the first and second quartiles did not benefit much from the training program (most likely due to ceiling effects). Drivers in the 
third quartile benefited from training, although these benefits diminished over time. By contrast, drivers in the fourth quartile demonstrated substantial benefits throughout the post-training interval. Thus, a clear pattern emerges with respect to the drivers that benefit most from training: those with the poorest pre-training performance benefit most from the fuel management program.

Another issue of importance is how driver characteristics such as age and job tenure influence the effectiveness of the training program. We performed analyses in which drivers were sorted into quartiles based on their age or based on their years of tenure with the company. Statistical analysis failed to reveal any reliable effects of age or tenure, indicating that the fuel management training program is of significant value for drivers with a wide range of ages and prior experience.

Finally, we performed an analysis comparing the subset of drivers $(n=10)$ who changed vehicles shortly after training with the drivers $(n=30)$ who drove the same vehicle over the 6 -month posttraining interval. This analysis is of interest because it tests to see if the training program taught drivers a generalized skill that would transfer from one vehicle to another. Figure 6 presents the data for these two groups of drivers. Both same-vehicle and switched-vehicle groups benefited from training, $\mathrm{F}(1,38)=8.0, \mathrm{p}<.01$. Most importantly, whether or not drivers changed vehicles did not significantly alter the training effect (i.e., the interaction of training with vehicle change was not significant, $\mathrm{p}<.4$ ), indicating that the skills acquired during the fuel management program transferred from one vehicle to another. That is, the training program taught drivers a generalized skill to optimize shifting and that skill transferred when the driver changed vehicles.

In summary, these data provide strong validation for the GE Driver Development fuel management program. The benefits of training are significant, appear to be sustained over time, and tend to benefit most those drivers whose performance was initially below the median on fuel efficiency. Drivers' age and tenure did not affect the efficiency of the training. Cost-benefit analysis suggests that simulator-based training provides a very effective means of reducing operating expenses for commercial trucking, particularly for less skilled drivers.

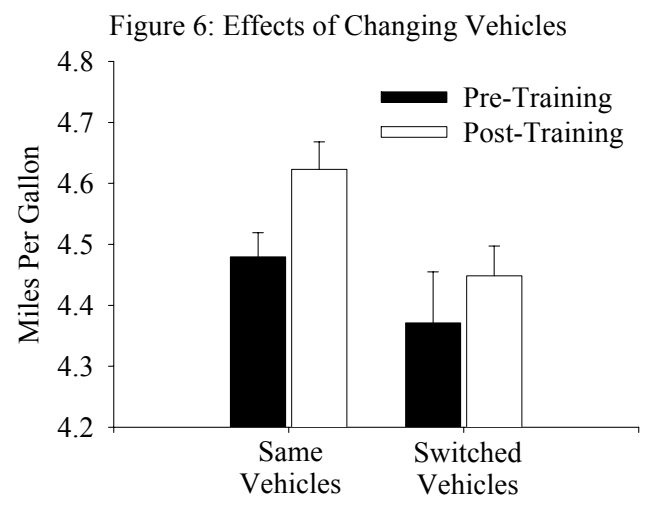

